\title{
EL PROCESAMIENTO DE LA INFORMACIÓN DE LAS PERSONAS MAYORES Y SU ACTITUD HACIA EL ANUNCIO
}

Estrada, M.

Sánchez, J.

Fandos, J.C.

Universitat Jaume I de Castellón (Spain)

estrada@emp.uji.es

jsanchez@emp.uji.es

jfandos@emp.uji.es

Material original autorizado para su primera publicación en la revista académica REDMARKA. Revista Digital de Marketing Aplicado

https://doi.org/10.17979/redma.2011.01.07.4721

Recibido: 11 Julio 2011

Aceptado 9 Diciembre 2011

\section{RESUMEN:}

El envejecimiento poblacional es un hecho de gran transcendencia socioeconómica con importantes repercusiones empresariales. Es importante el análisis del consumidor mayor y de sus particularidades para la consecución de una efectiva comunicación publicitaria. En este trabajo se analizan los antecedentes de la actitud ante el anuncio de las personas mayores y sus consecuencias sobre la actitud hacia la marca y la intención de compra en condiciones de baja implicación con el anuncio. Sobre una muestra constituída por 509 sujetos se confirma el modelo general planteado y se defiende que las personas mayores utilizan conjuntamente las dos rutas de persuasión en el procesamiento cognitivo del anuncio.

Palabras clave: personas mayores, actitud hacia el anuncio, baja implicación 


\section{ABSTRACT:}

PROCESSING INFORMATION OF ELDER PEOPLE AND THEIR ATTITUDE TOWARDS ADS

Population ageing is a phenomenon of great socio-economic importance with major consequences for business. Analysis of older consumers and of their peculiarities is important for achieving effective advertising communication. This study analyses the antecedents of older people's attitude to the ad and its effects on the brand attitude and purchasing intention in conditions of low involvement with the ad. From a sample consisting of 509 individuals, the general model posited is confirmed and older people are found to use both persuasion paths jointly in the cognitive processing of the ad.

Keywords: older people, attitude to the ad, low involvement 


\section{INTRODUCCION}

En los últimos años, desde diversas áreas como la sociología, la psicología, la economía y el marketing (Bermejo, 2008; Catterall y Maclaran, 2001; Goldberg, 2009; Ramos, 2007) se ha llamado la atención sobre la progresiva incorporación de la generación del "baby boom" (nacidos entre 1946 y 1964) a la tercera edad, apelando a la necesidad de analizar las repercusiones que este fenómeno tendrá sobre la economía y el consumo.

Las personas mayores constituyen uno de los segmentos más atractivos para las economías desarrolladas, ya que se trata de un colectivo cada vez más numeroso (en España las personas mayores de 65 años representan el 16.9 por ciento de la población), con una participación activa en la sociedad y con un poder adquisitivo igual o incluso más alto que el que disponen otros grupos más jóvenes (IMSERSO, 2006, 2008).

Desde los años 80 la literatura (Azofra, 1996; Grande, 1993, 1999, 2000, 2002) apela a la necesidad de investigar sobre la heterogeneidad (de salud, psicológica, social y de estilos de vida) y la particular idiosincrasia de este grupo (Bódalo, 2002). Existen muchos tipos de vejez y muchas maneras de "hacerse mayor" (Moschis y Mathur, 2006). El envejecimiento es un proceso de transformaciones biológicas, sociales, psicológicas, de estilos de vida, etc que tienen su reflejo en el procesamiento de la información publicitaria condicionando la actitud de las personas mayores. En este trabajo estudiaremos como el particular procesamiento de información de los mayores influye en la formación de la actitud hacia el anuncio teniendo consecuencias sobre las variables actitud hacia la marca e intención de compra en condiciones de baja implicación con el anuncio. Los resultados obtenidos pretenden orientar a las empresas en la logro de eficaces campañas publicitarias para/con este público ya que, en ocasiones, el desconocimiento de las peculiaridades de este colectivo merma la efectividad de su comunicación.

\section{REVISIÓN DE LA LITERATURA}




\subsection{Concepto actitud hacia el anuncio}

Las actitudes son constructos hipotéticos que se manifiestan en evaluaciones sumarias de objetos (uno mismo, otras personas, cosas, etc) en una dimensión bipolar que va de los positivo a lo negativo (agrado-desagrado; a favor-en contra) y son respuestas aprendidas (Zimbardo y Leippe, 1991). En su estructura se identifica: un componente afectivo, un componente cognitivo y un componente conductual. Distinguiendo, según su objeto, entre: la actitud hacia el producto (dentro de la cual se distingue entre actitud hacia la marca, modelo...) y la actitud hacia lo que rodea al producto (el anuncio, el canal de venta, el vendedor...) (Bermejo, 2008).

La complejidad del constructo actitud ha provocado durante mucho tiempo una falta de diferenciación entre la actitud hacia el anuncio y la actitud hacia la marca. En este sentido, notable es la aportación de Mitchell y Olson (1981) al ser los primeros autores en considerar el carácter independiente pero relacionado de ambos conceptos. La actitud hacia el anuncio supone la respuesta a un estímulo publicitario que ejerce una influencia sobre la actitud hacia la marca y la intención de compra (Bermejo, 2008; Mackenzie, Lutz y Belch, 1986; Mackenzie y Lutz, 1989). Los mecanismos de reacción hacia el estímulo publicitario pueden explicarse a partir de las relaciones causales que se plantean en los llamados modelos de actuación o respuesta publicitaria: el modelo AIDA, el de Lavidge y Steiner (1961), el de Batra y Ray (1986), etc. Concretamente en en este trabajo plantemos un modelo teórico que se basa en el de mediación dual (Mackenzie, Lutz y Belch, 1986) y lo completamos añadiendo la relación entre la actitud hacia el anuncio y la intención de compra (Figura 1).

Figura 1. Modelo teórico planteado

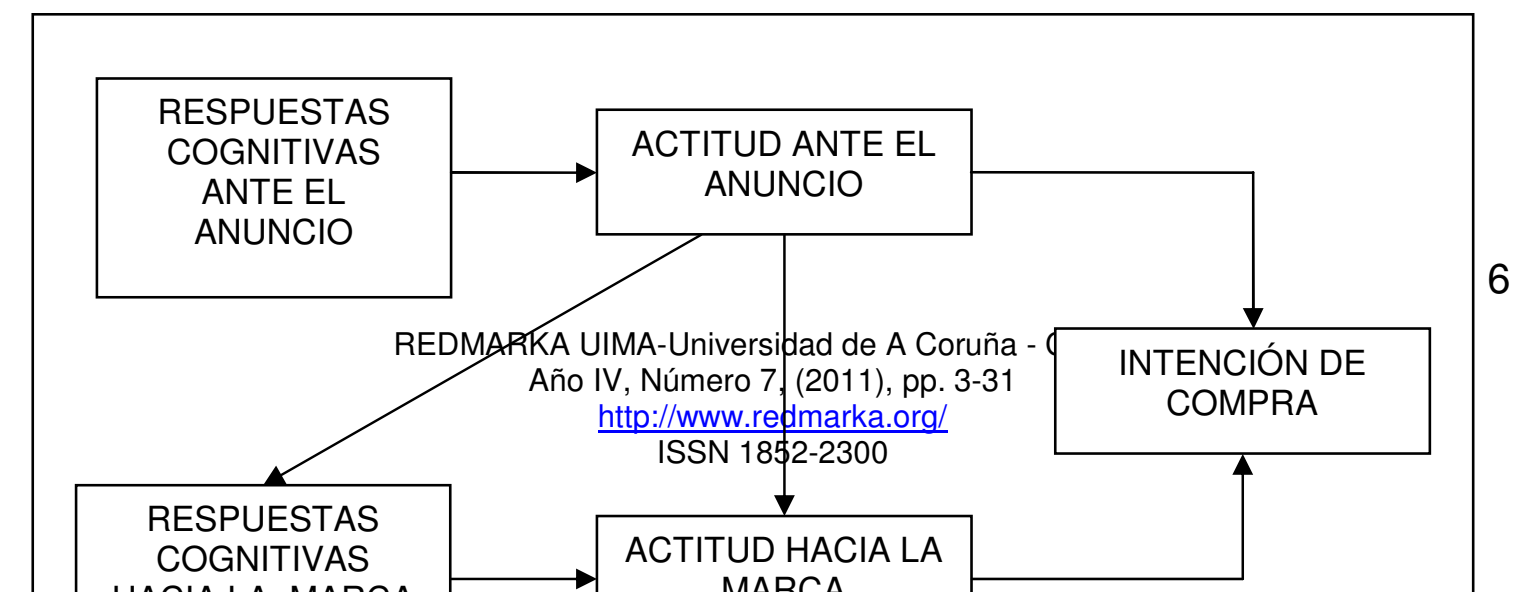


Defendemos que la existencia de un estímulo publicitario provoca unas respuestas cognitivas, unas creencias que, además de tener una influencia positiva en la actitud hacia el anuncio (Homer, 1990; Smith, 1993) también ejercen una función indirecta en la relación existente entre la actitud hacia el anuncio y la evaluación previa hacia la marca (Chattopadhyay y Basu, 1990). Asi mismo, la actitud hacia el anuncio influye sobre las respuestas cognitivas hacia la marca y la actitud hacia la marca (Mackenzie et. al, 1986; Mackenzie y Spreng, 1992). Por último, los anuncios más positivamente evaluados ejercen una mayor influencia sobre la intencionalidad de compra. A su vez, esta variable comportamental, aparece relacionada por la actitud hacia la marca (Bigné y Sánchez, 2001, 2003; Brennan y Bahn, 2006).

La actitud hacia el anuncio y, por ende, las relaciones planteadas por el modelo se encuentran condicionadas por las variables internas (la edad, la inteligencia, la capacidad de procesamiento de la información, la autoestima, el estado de ánimo, etc) (Dutta-Bergman y Youn, 1999; Briñol et. al. 2004; Rhodes y Wood, 1992) y las variables externas (el contexto, el color, la composición el grafismo, etc). A las mismas se suman, entre otras, las actitudes previas, la habilidad general, la predisposición, el nivel socio-económico, la experiencia, la implicación y la capacidad de procesar la información (Bermejo, 2008; Estrada, et. al. 2010; Walsh y Mitchell, 2005). Las dos últimas variables adquieren especial relevancia en el caso de las personas mayores dadas la particularides de este colectivo. Los actitud hacia el anuncio y sus consecuencias pueden manifestarse de forma diferente en función del nivel de implicación y del procesamiento de la información (Bigné y Sánchez, 2001). Asi pues en este 
trabajo analizaremos el efecto moderador que sobre las relaciones planteadas tiene la implicación con el anuncio de las personas mayores.

\subsection{Implicación}

Los primeros estudios sobre el concepto implicación se encuentran en el campo de la sociopsicología, remontándose a los trabajos de Sherif y sus coautores (Sherif y Sherif, 1967). Sin embargo, la mayoría de investigaciones atribuyen a Krugman (1965) el origen y análisis de la implicación dentro del marketing. A partir de este estudio, la implicación se convierte en objeto de atención por parte de los investigadores en el campo del comportamiento del consumidor (Bigné y Sánchez, 2001; McColl-Kennedy y Fetter, 2001; Ortigueira y Vázquez, 2005; Warrington y Shim, 2000). Cuando hablamos de la implicación, en general, nos referimos al nivel de relevancia personal y de motivación individual para esforzarse y comprometerse en el proceso (Batra y Stayman, 1990).

Se ha estudiado el nivel de implicación desde varias perspectivas la implicación con el producto (Chartes y Pettigreus, 2006), con la marca (Aurefeille et. al. 2002), con los medios de comunicación (Krugman, 1965), con el cliente (Lagrosen, 2005), con el problema (Ibañez, et. al. 2007), con el anuncio (Olson y Thjømøe, 2003), etc.

Con respecto a la implicación con el anuncio ésta se ha definido como el grado de motivación que tiene el sujeto estando inducido por los estímulos de un anuncio en particular (Batra y Ray, 1985). En la implicación con el anuncio se distingue entre implicación con el contenido y la implicación con la ejecución (Gutiérrez y Royo, 2001). La implicación con el contenido del mensaje viene determinada por el procesamiento que realizan las personas de la información sobre los atributos del producto anunciado (Gutiérrez y Royo, 2001). La implicación con la ejecución refleja la cantidad de esfuerzo realizado por los consumidores para procesar aspectos del contexto del anuncio, es decir, elementos del anuncio no relacionados con el mensaje (la música, la apariencia de los personajes, el ambiente creado, etc) (Bigné y Sánchez, 2001; Gutiérrez y Royo, 2001). 
La implicación debe ser conceptuada también como un factor de intensidad (Mitchell, 1979). Por intensidad este autor considera la cantidad de atención prestada a determinados aspectos del anuncio. En la mayoría de los casos este factor es operacionalizado como alta (completa atención) o baja implicación (atención limitada). La noción de intensidad está, según el modelo ELM (Elaboration Likelihood Model) (Petty, Cacioppo y Schuman, 1983), caracterizada en términos de procesamiento central o periférico, donde el procesamiento central representa una implicación alta y el periférico una baja implicación. Originariamente el modelo afirma que, cuando el nivel de interés o identificación es más alto, se utilizará la ruta central, en cambio, la ruta periférica convendrá cuando su participación sea escasa.

En la ruta central de persuasión, los sujetos están muy identificados con el producto o mensaje. Se sienten más proactivos ante la información que les llega sobre el producto, se muestran más motivados que en la ruta periférica para prestar atención a la información central relacionada con el producto (Capon y Lutz, 1983; Wu, 2001). Dada su gran participación, tienden a comprender esta información en niveles más profundos y complejos, conectándola con experiencias y conocimientos anteriores. Cuando la ocurrencia o likelihood en la elaboración es alta se originan creencias relacionadas con el anuncio, actitudes favorables hacia la marca y hacia la intención de compra (Briñol y Petty, 2006). En alta implicación, al realizarse un procesamiento cognitivo profundo, se incrementa la memoria y las posibilidades de recuerdo (Garder, Mitchell y Russo, 1985). La utilización de la ruta central de persuasión no sólo depende de la existencia de una gran motivación en el procesamiento de la información, sino de la disposición de la habilidad en el procesamiento del mensaje por parte del sujeto (Te'eni-Harari, Lampert and Lehman-Wilzig, 2007).

\subsection{El procesamiento de la información y las personas mayores}

Las personas mayores, como consecuencia de déficits cognitivos asociados a la edad, reducen su capacidad de búsqueda de información, tienden a búsquedas superficiales, inspeccionando sólo unas pocas alternativas para 
encontrar una solución aceptable, tienen tendencia hacia el uso de la ruta periférica (Cole y Balasubramunian, 1993). La edad, las distracciones, la ansiedad, la utilización de medios de comunicación inadecuados, la naturaleza del mensaje, etc, son algunos de los ruidos que contribuyen en la alteración de esta habilidad (Briñol y Petty, 2006; Te'eni-Harari, Lampert y Lehman-Wilzig, 2007).

Las personas mayores tienden a fijarse en algunos aspectos periféricos (las fotografías de un anuncio, las imágenes familiares, el atractivo de la fuente, la credibilidad) por su valor de entretenimiento (Spotts, 1994) y, lo que piensan sobre los aspectos periféricos ajenos al producto, podría quedar integrado en una actitud favorable hacia el anuncio. Más tarde, cuando se presente la ocasión de realizar una compra y las personas mayores deban evaluar algunas marcas, activarán esos significados conexos con el anuncio para crear una actitud hacia la marca o generar la intención de compra (Sengupta, Goodstein y Boninger, 1997).

Un tema muy controvertido en torno al análisis del ELM es el que hace referencia a la falta de explicación por parte del modelo de una posible interacción entre los procesos central y periférico (Crano y Prislin, 2005; Kim, 2003). Según Eagly y Chaiken (1993), ambos sistemas de procesamiento pueden ocurrir a la vez.

Los mayores pueden estar más atentos que los jóvenes a los elementos periféricos usados en el anuncio $\mathrm{y}$, por tanto, son más sensibles a su descripción en el anuncio (Loken, 2006; Yoon, 1997). Sin embargo, algunas investigaciones han polemizado sobre la posibilidad de que elementos periféricos puedan ser procesados centralmente en algunas ocasiones. De lo que se deduce que, en situaciones de baja implicación, los mayores pueden ser más proclives que los jóvenes a procesar los elementos periféricos por la ruta central con el fin de reducir sus déficits en la capacidad de procesamiento (Spotts, 1994). Esto implicaría que, en el proceso de formación y cambio de actitudes, se podría producir conjuntamente por la ruta central y periférica. Por lo tanto, éstas no solamente no son dos rutas mutuamente excluyentes, sino 
complementarias, dado que ambas resultan significativas en la formación y/o cambio de actitud hacia el anuncio y la marca en condiciones de baja implicación (Falces et. al. 2001; Homer, 1990; MacKenzie, Lutz y Blech, 1986; Miniard, Bhatla y Rose, 1990; Rodríguez, Del Barrio y Castañeda, 2006).

Llegado a este punto y revisada la literatura pertinente en este trabajo planteamos un modelo que defiende 6 hipótesis de trabajo a aceptar en la parte empírica de esta investigación (Tabla 1).

Tabla 1. Hipótesis de trabajo.

$\mathrm{H}_{1}$ : En condiciones de baja implicación con el anuncio, las respuestas cognitivas de las personas mayores ante el anuncio influyen más en su actitud hacia el mismo que en alta implicación.

$\mathrm{H}_{2}$ : En condiciones de baja implicación, la actitud hacia el anuncio de las personas mayores influye más en las respuestas cognitivas hacia la marca que en alta implicación.

$\mathrm{H}_{3}$ : $\quad$ En condiciones de baja implicación, las respuestas cognitivas hacia la marca de las personas mayores influyen más en su actitud hacia la marca que en alta implicación.

$\mathrm{H}_{4}$ : $\quad$ En condiciones de baja implicación, la actitud hacia el anuncio de las personas mayores influye más en su actitud hacia la marca que en alta implicación.

$\mathrm{H}_{5}$ : En condiciones de baja implicación, la actitud hacia el anuncio de las personas mayores influye más en su intención de compra que en alta implicación.

$\mathrm{H}_{6:} \quad$ En condiciones de baja implicación, la actitud hacia la marca de las personas mayores influye más en su intención de compra que en alta implicación.

\section{METODOLOGÍA}


En el diseño de esta investigación se han seleccionado al azar cuatro anuncios publicados en revistas especializadas dirigidas al público mayor. Durante el transcurso de un prestest con cuatro anuncios se han seleccionado los anuncios de pan de molde y de bebida deportiva, al ser éstos con los que sujetos se han mostrado más bajamente implicados (Figura 2). Este pretest también ha permitido realizar las rectificaciones oportunas a nivel gramatical para conseguir una mejor comprensión del cuestionario.

En la elección del medio a utilizar nos hemos decantado por el impreso al ser el más efectivo para dirigirse a las personas mayores ya que permite controlar mejor la información, tomarse el tiempo necesario para asimilarla, organizarla, seleccionar lo que le interesa, guardar los ejemplares e incluso transferirlos (Grande, 2000; Schewe, 2001; Garcillán y Grande, 2002).

Figura 2. Anuncios utilizados
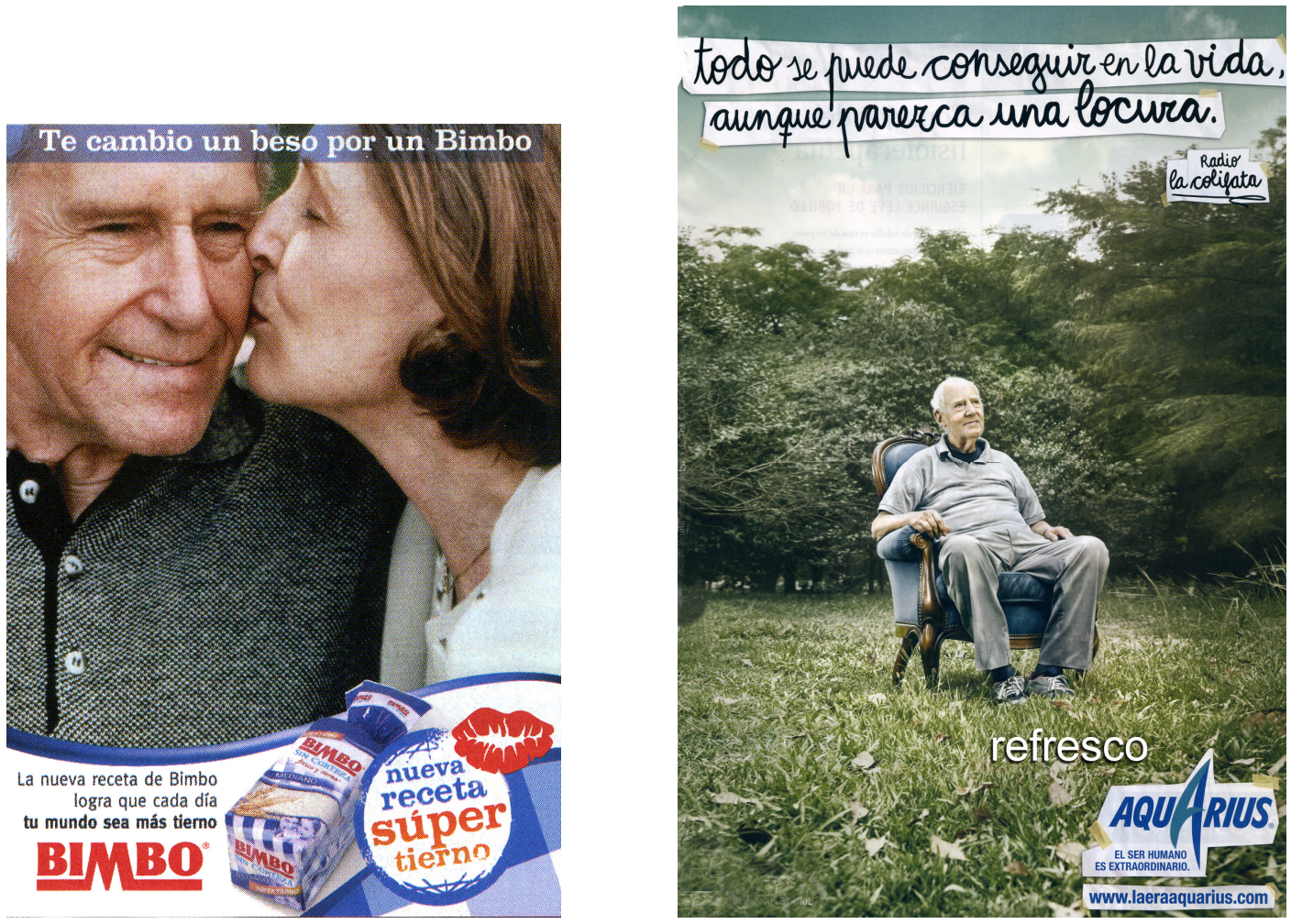

\subsection{Muestra}


En la obtención de datos, la población objeto de estudio asciende a un total de 751,762 personas mayores de 65 años residentes en el levante español, de las cuales en el municipio de Castellón hay empadronadas 89,289, en el de Valencia 383,130 y en el de Alicante 279,343 (último Censo de Población y Vivienda, 2001, en www.ine). Se ha utilizado la edad oficial de la jubilación, 65 años, como punto de referencia para estudiar a las personas mayores.

El tamaño teórico de la muestra originariamente ha sido de 512 pero tras la realización de un análisis cluster en el que se eliminan los casos atípicos se ha reducido a 509 sujetos. Para la distribución por edad, género y municipio de la muestra se han tomado como referencia los datos del Censo de Población y Vivienda, 2001 (www.ine.es) utilizándose como criterio de distribución la realización de un mínimo de 100 encuestas personales por municipio (Tabla 2). El muestreo ha sido estratificado con afijación proporcional en función de la distribución de la población según las variables de edad, género y hábitat y supone un error muestral del \pm 4.4 por ciento para un nivel de confianza del 95.5 por ciento y un $\mathrm{p}=\mathrm{q}=0.5$

Tabla 2. Distribución de la muestra según criterios de segmentación sociodemográfica

Distribución de la muestra por edad

Años

$\mathrm{N}$

Hombres

Mujeres

65 a 74

303

145

Más de 74

206

100

Total

509

264

245

Distribución de la muestra por nivel de estudios

Nivel de estudios 
Tanto por cierto

Primarios

77.8

Bachiller y universitarios

22.2

Total

100

Distribución de la muestra por la clase social de pertenencia

Clase social

Tanto por ciento

Alta

5.5

Media

59.3

Baja

5.2

Total

100

Para la obtención de la muestra se ha recurrido al diseño de una base de datos formada por el conjunto de CEAMS (centros especializados de atención a las personas mayores) de la Comunidad Valenciana y de asociaciones de personas mayores. La selección de las unidades a encuestar se ha realizado en centros y asociaciones ubicados en barrios con diferente nivel adquisitivo y cultural para favorecer la heterogeneidad de la muestra.

\subsection{Cuestionario}

La información se ha recogido a través de un cuestionario estructurado formado por seis partes destinadas a valorar cada variable objeto de estudio. 
Para la medición de las variables se ha recurrido a aquellas escalas más utilizadas en la literatura.

En el caso concreto de la actitud hacia el anuncio los pares de ítems utilizados han sido: es muy malo/es muy bueno, no me ha gustado nada/me ha gustado mucho, no es nada interesante/es muy interesante, tengo una opinión muy desfavorable/tengo una opinión muy favorable y es muy desagradable/es muy agradable (Beerli y Martín, 1999; Curlo y Ducoffe, 1998; Kempf y Smith, 1998).

Para valorar las respuestas cognitivas hacia el anuncio se han escogido los pares de ítems: es poco informativo/es muy informativo, es poco convincente/es muy convincente, es poco persuasivo/es muy persuasivo, tiene unos argumentos muy débiles/tiene unos argumentos muy fuertes y es poco creíble/es muy creíble (Olney, Holbrook y Batra,1991).

Con respecto a la actitud hacia la marca, se han utilizado los ítems: es muy mala/es muy buena, no me gusta nada/me gusta mucho, tengo una opinión muy desfavorable/tengo una opinión muy favorable, es muy desagradable/es muy agradable y considero que tiene muy poco prestigio/considero que tiene mucho prestigio (Beerli y Martín, 1999; MacKenzie y Spreng, 1992).

Para valorar las respuestas cognitivas hacia la marca se han utilizado los siguientes pares de ítems: tiene mucha calidad/ tiene poca calidad, tiene buen precio/tiene mal precio, tiene buena apariencia/tiene mala apariencia, ofrece una gran garantía/ ofrece pocas garantías (Sánchez, 1999).

En relación a la intención de compra se ha recurrido a los ítems: seguro que no, poco probable, no lo sé, probablemente sí, seguro que sí (Mackenzie y Spreng, 1992; Yoon et. al. 1995).

Respecto a a medición de la variable implicación con el anuncio, con objeto de diferenciar a los sujetos más altamente implicados de los más bajamente implicados, se utilizaron los ítems: en la foto, en el texto, en ninguna de las dos cosas, en ambas cosas a la vez. Posteriormente se consideraría que, aquellos sujetos que contestaban afirmativamente a una de las tres primeras opciones, revelaban un menor interés por el anuncio mientras que aquellos que 
contestaban afirmativamente a la cuarta cuestión revelaban un mayor interés (Hawkins y Hock, 1992; Sánchez, 1999).

En cuanto a las alternativas de respuesta se ha optado por la combinación de una escala Likert (de 5 puntos) y una escala Kunin. Este tipo de escalas son muy fáciles de recordar, no requieren conocimientos especiales y generalmente no fatigan (Jayanti, McManamon y Whipple, 2004) lo que supone una ventaja esencial a la hora de diseñar un cuestionario dirigido a personas mayores de 65 años.

\section{ANÁLISIS DE DATOS}

En el análisis de datos se ha procedido a determinar la calidad de las escalas de medida utilizadas, para ello, se ha realizado un análisis factorial confirmatorio a través de la técnica de modelos de ecuaciones estructurales y del uso del programa estadístico LISREL 8.3.

Como consecuencia de la utilización de las mismas escalas de medición para los dos anuncios analizados, con objeto de generalizar, se ha realizado de forma conjunta el estudio de la dimensionalidad, fiabilidad y validez de las escalas. Los resultados muestran para los modelos obtenidos una adecuada bondad en el ajuste del modelo debido a que los valores alcanzados por la probabilidad asociada a la chi-cuadrado han sido superiores a 0.05 (Jöreskog y Sörbom, 1996), además el índice de bondad de ajuste (GFI) y el índice de ajuste comparativo (CFI) presentan valores no inferiores al recomendado 0.90. Por último, el valor del error de aproximación cuadrático medio (RMSEA) es inferior al límite máximo recomendado 0.08 .

La validez convergente ha quedado demostrada al ser las cargas factoriales significativas y superiores a 0.6 (Bagozzi y Yi, 1988) y la varianza extraída promedio AVE para cada uno de los factores superior a 0.5 (Fornell y Larcker, 1981). Con respecto a la validez discriminante ésta ha quedado comprobada al ser la raiz cuadrada del AVE superior a todas las correlaciones entre los factores (Fornell y Larcker, 1981). 
La fiabilidad compuesta ha quedado ratificada al ser los índices de cada una de las dimensiones obtenidas superiores a 0.6 (Bagozzi y Yi, 1988).

Una vez estudiadas las propiedades psicométricas de las escalas de medida se ha dividido a la muestra en dos grupos, un primer grupo constituidos por los sujetos según altamente implicados con el anuncio (214 sujetos) y un segundo grupo con los sujetos bajamente implicados con el anuncio (295 sujetos), realizándose, mediante la técnica ANOVA, un análisis descriptivo de la muestra en función del nivel de implicación con el anuncio. En este análisis se observaron la existencia de diferencias significativas entre alta y baja implicación.

Tabla 3. Análisis ANOVA de la muestra según la implicación con el anuncio

Alta implicación

implicación

$$
\text { pan de molde bebida deportiva }
$$

pan de molde

bebida deportiva

\begin{tabular}{llll}
\hline R.C.A. & $3.44^{*}$ & $3.03^{\star}$ &
\end{tabular}

\section{$2.95^{\star}$}

\section{A.A}

$3.70^{*}$

$3.68^{*}$

$3.34^{*}$

$3.20^{*}$

\begin{tabular}{llll}
\hline R.C.M. & $3.87^{*}$ & $3.72^{*}$ & $3,49^{*}$ \\
$2.92^{*}$ & & & \\
\hline
\end{tabular}

\begin{tabular}{lccc}
\hline A.M. & $4.04^{*}$ & $3.66^{*}$ & $3.63^{*}$ \\
$2.92^{*}$ & & & \\
\hline I.C & $3.74^{*}$ & $3.72^{*}$ & $3,51^{*}$ \\
$2.92^{*}$ & & & \\
\hline
\end{tabular}

Note. *diferencias significativas 
R.C.A.: respuestas cognitivas ante el anuncio; A.A.: actitud ante el anuncio; R.C.M.: respuestas cognitivas ante la marca; A.M.: actitud hacia la marca; I.C.: intención de compra

Posteriormente se ha procedido a verificar el cumplimiento de las hipótesis a través de un análisis de la relaciones causales en función del nivel de implicación con el anuncio aplicando para ello un análisis path (Jöreskog y Sörbom, 1996). Para este caso se diferenció entre baja y alta implicación creándose 4 casos diferentes que fueron el resultado de la creación de 2 escenarios (alta y baja implicación) para cada uno de los dos anuncios considerados. Se realizaron pues, en este caso, hasta un total de 4 modelos que mostraron un ajuste del modelo adecuado ya que la probabilidad asociada al estadístico chi-cuadrado ha sido superior a 0.05 para los dos anuncios analizados.

Tabla 4. Análisis de las relaciones causales en baja implicación ${ }^{1}$

\begin{tabular}{|c|c|c|}
\hline bebida deportiva & pan de & molde \\
\hline $\begin{array}{l}\text { R.C.A. } \rightarrow \text { A.A. } \\
0.90(25.66)\end{array}$ & & $0.88(19.71)$ \\
\hline $\begin{array}{l}\text { A.A. } \rightarrow \text { R.C.M. } \\
0.54(9.69)\end{array}$ & & $0.50(8.29)$ \\
\hline $\begin{array}{l}\text { R.C.M. } \rightarrow \text { A.M. } \\
0.89(33.26)\end{array}$ & & $0.79(24.03)$ \\
\hline $\begin{array}{l}\text { A.A. } \rightarrow \text { A.M. } \\
0.05(2.05)\end{array}$ & & $0.15(4.76)$ \\
\hline $\begin{array}{l}\text { A.A. } \rightarrow \text { I.C. } \\
0.05(2.05)\end{array}$ & & $0.16(4.76)$ \\
\hline $\begin{array}{l}\text { A.M. } \rightarrow \text { I.C. } \\
0.75(19.06)\end{array}$ & & $0.60(12.95)$ \\
\hline
\end{tabular}




\section{AJUSTE DEL MODELO}

pan de molde : Chi-square $=2.94 ; \mathrm{gl}=4 ; \mathrm{p}=0.568 ; \mathrm{RMSEA}=0.000 ; \mathrm{GFI}=1$; $\mathrm{AGFI}=0.99$

bebida deportiva: Chi-square $=7.04 ; \mathrm{gl}=3 ; \mathrm{p}=0.070 ; \mathrm{RMSEA}=0.069 ; \mathrm{GFI}=0.99$; $A G F I=0.95$

Note. ${ }^{1}$ Coeficientes del anàlisis "path" (significatividad).

R.C.A.: respuestas cognitivas ante el anuncio; A.A.: actitud ante el anuncio; R.C.M.: respuestas cognitivas ante la marca; A.M.: actitud hacia la marca; I.C.: intención de compra

Tabla 5. Análisis de las relaciones causales en alta implicación ${ }^{1}$

pan de molde

bebida deportiva

\begin{tabular}{lc}
\hline R.C.A. $\rightarrow$ A.A. & $0.78(17.87)$ \\
$0.80(19.77)$ & \\
\hline A.A. $\rightarrow$ R.C.M. & $0.44(6.32)$ \\
$0.47(8.15)$ & \\
\hline R.C.M. $\rightarrow$ A.M. & $0.73(16.06)$ \\
$0.81(20.91)$ & \\
\hline A.A. $\rightarrow$ A.M. & $0.13(3.19)$ \\
$0.09(2.31)$ & \\
\hline A.A. $\rightarrow$ I.C. & $0.13(3.19)$ \\
$0.13(2.16)$ &
\end{tabular}

A.M. $\rightarrow$ I.C.

$0.39(6.62)$

$0.45(7.55)$

\section{AJUSTE DEL MODELO}

pan de molde: Chi-square $=9.22 ; \mathrm{gl}=4 ; \mathrm{p}=0.055 ; \mathrm{RMSEA}=0.078 ; \mathrm{GFI}=0.98$; 
AGFI=0.94

bebida deportiva: Chi-square $=4.10 ; g l=3 ; p=0.251 ; \mathrm{RMSEA}=0.041 ; \mathrm{GFI}=0.99$; AGFI=0.96

Nota. ${ }^{1}$ Coeficientes del anàlisis "path" (significatividad).

RCA: Respuestas cognitivas ante el anuncio; AA: Actitud ante el anuncio; RCM: Respuestas cognitivas ante la marca; AM: Actitud hacia la marca; IC: Intención de compra

\subsection{Resultados}

El análisis de los datos ha demostrado que con respecto a la relación planteada sobre la influencia que ejercen las respuestas cognitivas ante el anuncio sobre la actitud hacia el anuncio los valores tomados en el caso de baja implicación con el anuncio (pan 0.88; bebida 0.90) han sido superiores a los tomados en alta implicación (pan 0.78; bebida 0.80). Esto nos ha permitido aceptar la hipótesis 1 de nuestro estudio. Además los resultados han mostrado que, los valores obtenidos en esta relación, tanto en alta como en baja implicación, han sido mayores si los comparamos con el resto de valores alcanzados para el resto de relaciones causales. Esto indica la importancia que tiene la dimensión cognitiva en el procesamiento mental que realizan las personas mayores y su posterior actitud hacia el anuncio (Bódalo, 2002). Dichos hallazgos coinciden con las investigaciones que mantiene que, en bajo nivel de implicación, el afecto no es identificado (Crano y Prislin, 2005).

Con respecto a la influencia ejercida por la actitud hacia el anuncio en las respuestas cognitivas hacia la marca observamos que, en baja implicación (pan 0.50 , bebida 0.54 ), los valores son superiores a los de alta implicación (pan 0.44 , bebida 0.47 ), por lo que se acepta la hipótesis 2 de nuestro estudio. En este sentido resulta interesante observar que, tal y como defienden los trabajos de Olson y Thjømøe (2003), los valores para baja implicación en la relación de la actitud hacia el anuncio y de las respuestas cognitivas hacia la marca son muy superiores a los obtenidos en la relación entre la actitud hacia el anuncio y la actitud hacia la marca. 
En referencia a la hipótesis 3 , los valores obtenidos del análisis de la relación entre las respuestas cognitivas hacia la marca y la actitud hacia la marca ha observado que, en baja implicación (pan 0.79; bebida 0.89) son superiores a los de alta (pan 0.73; bebida 0.81), por lo que se acepta el planteamiento defendido en la hipótesis de trabajo. De lo que se deduce que, en baja implicación, un importante antecedente de la actitud hacia la marca son las creencias hacia la marca (Lord, Lee y Sauer, 1995).

Con repecto a hipótesis 4, ésta se acepta parcialmente, al demostrarse empíricamente que, con respecto a la relación entre la actitud hacia el anuncio y la actitud hacia la marca, los valores obtenidos en baja implicación (pan 0.15; bebida 0.05 ) son superiores a los de alta (pan 0.13 ; bebida 0.13 ) tan sólo en el caso de pan de molde pero no en el caso de bebida deportiva.

En relación a la influencia ejercida por la actitud hacia el anuncio en la intención de compra (Homer, 1990; Yoon et. al. 1995) los valores obtenidos en baja implicación (pan 0.16 , bebida 0.05 ) resultaron superiores a los obtenidos en alta implicación (pan 0.13 , bebida 0.13 ) únicamente en el caso del pan de molde. Esto supone que la hipótesis 5 de nuestro estudio se acepta tan sólo parcialmente. Mientras que, los resultados obtenidos en la bebida deportiva, secundan los trabajos realizados en personas más jóvenes al demostrar que, en baja implicación, la actitud hacia el anuncio influye menos en la intención de compra que en alta implicación (Petty, Cacioppo y Schumann, 1983; Te'eniharari, Lampert y Lehman-Wilzig, 2007). Por otro lado, según los valores obtenidos, tanto en el caso de baja como de alta implicación, la influencia de la actitud hacia el anuncio en la intención de compra de las personas mayores no es excesivamente elevada. La explicación a este hecho reside en que, los mayores, en su proceso de búsqueda de información y posterior intención de compra, suelen recurrir - en orden de importancia- a la opinión de los familiares, su experiencia y conocimiento, amigos, vendedores y por último la información publicitaria (Grande, 1993; Ramos, 2007).

Por último, con respecto al grado de cumplimiento de la hipótesis 6 se acepta que, en baja implicación (pan 0.60; bebida 0.75 ), es superior la influencia de la 
actitud hacia la marca en la intención de compra que en alta implicación (pan 0.39; bebida 0.45). Las personas mayores buscan entre sus recuerdos de marca, en sus propias experiencias, con el fin de obtener la información necesaria para proceder a la compra (Cole y Balasubramanian, 1993).

\section{CONCLUSIONES}

En nuestra investigación, se demuestra que, en el caso de las personas mayores, existe una influencia de la actitud hacia el anuncio en la actitud hacia la marca y la intención de compra en condiciones de baja implicación con el anuncio. El modelo teórico planteado y posteriormente confirmado se basa en el modelo de mediación dual pero introduciendo la relación entre la actitud hacia el anuncio y la intención de compra que el modelo original no se planteaba (Lord, Lee y Sauer, 1995; Phelps y Hoy, 1996; Smith, 1993). modelo de mediación dual es el que mejor representa el comportamiento del consumidor tanto en situaciones de alta como de baja implicación (Homer, 1990), además dicho modelo introduce un elemento no considerado en el ELM: tanto la ruta central como la periférica pueden actuar conjuntamente en el proceso de formación o cambio de actitudes.

En las personas mayores existe una tendencia hacia la baja implicación y hacia el uso de la ruta periférica. Sin embargo, tal y como se demostró empíricamente, los mayores, con el fin de comprender el significado del mensaje publicitario, están dispuestos a utilizar las dos rutas de persuasión.

Esta afirmación secunda las investigaciones que defienden la tendencia que tienen las personas mayores hacia la baja implicación (Yoon, 1997). Los mayores, debido a sus características personales, a unos imaginarios sociales asociados al deterioro del paso de los años y a una reducción considerable de los espacios e intereses vitales, tienden a la selección y recorte de los estímulos ambientales. Esto, junto a los déficits cognitivos asociados a la edad, explica las bajas expectativas de logro que sostienen en relación a sus posibilidades en el procesamiento de la información (Schacter, 2003). A esto se le une una falta de interés por los productos presentados en los anuncios 
utilizados en nuestra investigación. Todo esto explica los valores superiores obtenidos entre las relaciones causales planteadas en baja sobre alta implicación.

De acuerdo con los postulados mantenidos en el ELM cabría esperar que, la tendencia hacia la baja implicación manifestada por los mayores, provocara un mayor interés o valoración hacia los elementos periféricos o afectivos, sin embargo, según los resultados de nuestro estudio, las personas mayores, necesitan de la racionalización del mensaje, de una búsqueda pragmática entre sus experiencias, expectativas, conocimientos y recuerdos que les ayuden a asociarlos con el anuncio y con la marca.

Los mayores generan estrategias fundamentales para compensar sus fallos cognitivos, dando importancia a la información recibida, buscando pistas para entender el anuncio (Bódalo, 2002; Grande, 1993, 1999; Ramos, 2007). Esto supone que, si bien en muchas ocasiones carecen de las habilidades para la realización de procesamientos cognitivos profundos, poseen la motivación necesaria para intentar mejorar sus déficits e intentar comprender el anuncio apelando a sus recuerdos y conocimientos. Los mayores, aún encontrándose en una situación de baja implicación, utilizan conjuntamente la ruta central y periférica con el fin de procesar la información contenida en el anuncio. Además, el encaje que produce la baja implicación provoca una mayor confianza en sus respuestas, en las propias creencias, aumentando las correlaciones entre pensamientos, actitud e intención (Briñol, Petty y Tormala, 2004).

Los anunciantes, si desean mejorar su efectividad con este público, deben utilizar en el diseño de sus anuncios elementos que puedan asociar a sus experiencias, combinar texto e imagen y utilizar argumentos racionales, sencillos y cortos. Sólo de esta forma podrán adapatarse a las particularidades de las personas mayores.

Por último comentar que en la realización de este estudio se han encontrado dificultades derivadas del proceso de envejecimiento lo que ha provocado ciertas dificultades: en la comprensión del cuestionario, la reducción de la 
capacidad de atención, comprensión y memorización de los encuestados. Por otro lado, debido a las restricciones presupuestarias, se ha limitado la localización de la muestra a una zona concreta de la geografía española. Además, los sujetos muestrales, pueden no ser representativos de todo el grupo de las personas mayores.

Como futuras líneas de investigación nos proponemos ampliar el estudio a otras zonas geográficas y utilizar otro tipo de productos (como productos de compra compleja) para observar los resultados obtenidos.

\section{REFERENCIAS BIBLIOGRÁFICAS}

Aaker, D.A. y Stayman, D. M. 1992. Implementing the concept of transformational advertising. Psychology \& Marketing, 9, 237-253.

Aurifeille, J.M., Quester, P.G., Lockshin, L. y Spawton, T. 2002. Global vs. internacional involvement-based segmentation a cross-national exploratory study. International Marketing Review, 19, 369-386.

Azofra, M.J. 1996. Comportamiento de los españoles ante las vacaciones. CIS, Madrid.

Bagozzi, R. y Yi, Y. 1988. On the evaluation of structural equation models. Journal of the Academy of Marketing Science, 16, 74-94.

Batra, R. y Ray, M.L. 1986. Affective responses mediating acceptance of advertising. Journal of Consumer Research, 13, 234-249.

Beerli, A. y Martín, J.D. 1999. Técnicas de Medición de la Eficacia Publicitaria. Editorial Ariel, Barcelona.

Bermejo, J. 2008. Actitud hacia el anuncio y tipología de respuestas publicitarias desde el mapa cultural del receptor.Pensar la Publicidad, 2, 93122.

Bigné, E. y Sánchez, J. 2001. Influencia del contenido informativo de la publicidad y de la implicación en un modelo de actitudes. Revista Europea de Dirección y Economía de la Empresa, 10, 85-102. 
Bigné, E. y Sánchez, J. 2003. Análisis del efecto moderador de la implicación con el producto y con el anuncio sobre la intensidad de las respuestas a la publicidad. Revista Española de Investigación de Marketing, 57-90.

Bódalo, E. 2002. Los mayores y el consumo, un estudio sociológico, Universidad de Murcia.

Brennan, I. y Bahn, K.D. 2006. Literal versus extended symbolic messages and advertising effectiveness: the moderating role of need for cognition. Psychology \& Marketing, 23, 273-295.

Briñol, P., Petty, R.E. y Tormala, Z. 2004: Self-validation of cognitive responses to advertisements. Journal of Consumer Research, 30, 559-572.

Briñol, P. y Petty, R. E. 2006. Fundamental processes leading to attitude change: implication for cancer prevention communications. Journal of Communication, 56, 81-104.

Capon, N. y Lutz, R.J. 1983. The marketing of consumer information. Journal of Marketing, 47, 108-12.

Catterall, M. y Maclaran, P. 2001. Body talk: questioning the assumptions in cognitive age. Psychology and Marketing, 18, 1117-1133.

Cole, C.A. (1986). Age differences in information processing understanding deficits in young and elderly consumers. Journal of Consumer Research, 13, 297-315.

Cole, C.A. y Balasubramanian, S. 1993. Age differences in consumers' search for information: public policy implications. Journal of Consumer Research, 20, 157-169.

Crano, W. D. y Prislin, R. 2005. Attitudes and persuasion. Annual Reviews Psychologic, 57, 345-374.

Curlo, E. y Ducoffe, R. 1998. Product use goals and attitudinal responses to ads. Journal of Current Issues of Research in Advertising, 20, 19-32.

Charters, S. y Pettigrew, S. 2006. Product involvement and the evaluation of wine quality. Qualitative Market Research: An International Journal, 9, 181-193. 
Chattopadhyay, A. y Basu, K. 1990. Humor in advertising: the moderating role of prior brand evaluation. Journal of Marketing Research, 17, 466-476.

Dutta-Bergman, M.J. y Youn, S. 1999. Profiling healthy eating consumers: a psychographic approach to social marketing. Social Marketing Quarterly, 5, 521.

Eagly, A. H. y Chaiken, S. 1993. Citado por Briñol, P., Petty, R.E. 2004. Selfvalidation processes: the role of thought confidence in persuasion. Ed. G.Haddock \& G.R. Maio. Contemporany perspectives on the psychology of attitudes, London.

Estrada, M.; Sánchez, J.; Moliner, M.A. and Fandos, J.C. (2010). Antecedentes y consecuencias de la actitud ante el anuncio en las personas mayores. Un análisis empírico. Innovar Journal, 20, 151-164.

Falces, C., Briñol, P., Sierra, B., Becerra, A. y Alier, E. 2001. Validación de la escala de necesidad de cognición y su aplicación al estudio del cambio de actitudes. Psicothema, 13, 622-628.

Fornell, C. y Larcker, D.F. 1981. Evaluating structural equations models with unobservable variables and measurement error. Journal of Marketing Research, 18, 39- 50.

Garcillan, M. y Grande, I. 2002. Factores determinantes de la capacidad de procesamiento de la información y su influencia en la creación del conjunto considerado implicaciones para la gestión de marketing. Cuadernos Aragoneses de Economía, 12, 111-130.

Gardner, M., Mitchell, A. y Russo, J. E. 1985. Low-involvement strategies for processing advertisements. Journal of Advertising, 14, 4-12.

Goldberg, M.E. 2009. Consumer decision marking and aging: A commentary from a policy/marketing perspective. Journal of Consumer Psychology, 19, 2428. 
Grande, I. 1993. Marketing Estratégico para la Tercera Edad: principios para atender a un segmento emergente. Escuela Superior de Gestión Comercial y Marketing (ESIC), Madrid.

Grande, I. 1999. Consumidores de la Tercera Edad ¿un segmento o muchos?. Distribución y Consumo, 45, 124-130.

Grande, I. 2000. Criterios de elección del establecimiento comercial por los consumidores de la tercera edad. Un estudio empírico. ESIC MARKET, 105, 114-127.

Grande, I. 2002. Influencia del cambio demográfico sobre la estructura del consumo, ahorro y endeudamiento de los hogares. Ekonomi Gerizan $X$, Federación de las Cajas de Ahorro Vasco-Navarras, 84-110.

Gutiérrez, A. Mª y Royo, M. 2001. El efecto del contenido emocional e informativo de la publicidad sobre la evaluación de los anunciantes en televisión. Revista Europea de Dirección y Economía de la Empresa, 10, 103118.

Hawkins, S.A. y Hoch, S.J. 1992. Low-involvement learning: memory without evaluation. Journal of Consumer Research, 19, 212-222.

Homer, P.M. 1990. The mediating role of attitude toward the ad: some additional evidence. Journal of Marketing Research, 27, 78-86.

Ibañez, J.A.; Montoro, F.J.; Sánchez, J. y Muñoz, F. (2007). Anàlisis de la efectividad del mensaje publicitario en la promoción de comportamientos de consumo sostenibles. Estudios sobre Consumo, 82, 9-17.

Instituto de Migraciones y Servicios Sociales (IMSERSO) 2006. Envejecer en España, IMSERSO. Madrid.

Instituto de Migraciones y Servicios Sociales (IMSERSO) 2008. Economía y personas mayores, , IMSERSO. Madrid.

Jayanti, R. K., McManamon, M.K. y Whipple, T.W. 2004. The effects of aging on brand attitude measurement. Journal of Consumer Marketing, 21, 264-273. 
Jöreskog, K.G. y Sörbom, D. 1996. LISREL 8: Structural Equation Modeling with the SIMPLIS Command Language. Scientific Software International, Chicago.

Kempf, D.S. y Smith, R.E. 1998. Consumer processing of product trial and the influence of prior advertising: a structural modeling approach. Journal of Marketing Research, 35, 325-338.

Kim, Y. 2003. Conceptualizing health campaign strategies through the level of involvement. International Journal, 8, 255-267.

Krugman, H.E. 1965. The impact of television advertising: learning without involvement. Public Opinion Quarterly, 29, 349-356.

Lagrosen, S. 2005. Customer involvement in new product development. a relationship marketing perspective. European Journal of Inovation Management, 8, 424-36.

Lavidge, R.J. y Steiner, G.A. (1961). A model for predicting measurement of advertising effectiveness. Journal of Marketing, 25, 59-62.

Loken, B. (2006). Consumer psychology: categorization, inferences, affect and persuasion", Annual. Review. Psychological, 57, 453-485.

Lord, K.R., Lee, M.S. y Sauer, P.L. 1995. The combined influence hypothesis: central and peripheral antecedents of attitude toward the aad. Journal of Advertising, 24, 73-85.

Mackenzie, S.B. y Lutz, R.J. 1989. An empirical examination of the structural antecedents of attitude toward the ad in an advertising pretesting context. Journal of Marketing, 53, 48-65.

Mackenzie, S.B., Lutz, R.J. y Belch, G.R. 1986. The role of attitude toward the ad as a mediator of advertising effectiveness: a test of competing explanations. Journal of Marketing Research, 23, 130-43.

Mackenzie, S.B. y Spreng, R.A. 1992. How does motivation moderate the impact of central and peripherical processing on brand attitudes and intentions?. Journal of Consumer Research, 18, 519-29. 
McColl-Kennedy, J.R. y Fetter, R.E. 2001. An empirical examination of the involvement to external search relationship in services marketing. The Journal of Services Marketing, 15, 2.

Mitchell, A. 1979. A Potencially Important Mediator of consumer Behavior. Advances in Consumer Research, 6, 191-96.

Michell, A.A. y Olson, J.C. 1981. Are product beliefs the only mediator of advertising effects on brand attitude?. Journal of Marketing Research, 18, 31832.

Miniard, P.W., Bhatla, S. y Rose, R.L. 1990. On the formation and relationship of ad and brand attitudes: an experimental and causal analysis. Journal of Marketing Research, 27, 290-03.

Moschis, G.P. y Mathur, A. 2006. Older consumer responses to marketing stimuli: the power of subjetive age. Journal of Advertising Research, 339-46.

Olney, T.J., Holbrook, M.B. y Batra, R. 1991. Consumer responses to advertising: the effects of ad content, emotions, and attitude toward the ad on viewing time. Journal of Consumer Research, 17, 440-53.

Olson, E. L. y Thjømøe, H. M. 2003. The effects of peripheral exposure to information on brand preference. European Journal of Marketing, 37, 243-55.

Ortigueira, M. y Vázquez, R. 2005. La implicación con el cliente: compilación teórica. Cuadernos de Gestión, 89-02.

Petty, R.E., Cacioppo, J.T. y Schumann, D. 1983. Central and periferical routes to advertising effectiveness: the moderating role of involvement, Journal of Consumer Reseach, 10, 135-46.

Phelps, J.E. y Hoy, M.G. 1996. The aad-ab-PI relationship in children: the impact of brand familiarity and measurement timing, Psychology \& Marketing, 13, 77-05.

Ramos, I. 2007. El estilo de vida de los mayores y la publicidad. Barcelona: Fundación La Caixa. 
Rhodes, N. y Wood, W. (1992). Self-steem and intelligence affect influenciability: the mediating role of message reception. Psychological Bulletin, $111,156-171$.

Rodríguez, M.A., Del Barrio, S. y Castañeda, J.A. 2006. Procesamiento diferencial entre la publicidad comparativa y la publicidad con famosos en condiciones de baja implicación. Proyecto de Investigación perteneciente al Plan Nacional de I+D (código IFD97-0306).

Sánchez, J. 1999. La actitud hacia el anuncio:el efecto moderador de la implicación y de la fuerza de los argumentos. Tesis doctoral, Universitat Jaume I, Castellón.

Schacter, D. (2003). Los siete pecados de la memoria. Ariel, Barcelona.

Schewe, C.D. 2001. Effective communication with our aging population. Business Horizons, 32, 19-25.

Sengupta, J., Goodstein, R.C. y Boninger, D.S. 1997. All cues not created equal: obtaining attitude persistence under low-involvement conditions. Journal of Consumer Research, 23, 351-61.

Sherif, M. y Sherif, C. 1967. Attitude, ego-involvement and change, John Wiley \& Sons, eds.; citado por Rodríguez, M. C., Cervantes, M. and González, A.M. 2006. El enfoque cognitivo en el análisis de la implicación temporal del consumidor con productos agroalimentarios de calidad. Revista Española de Investigación de Marketing. ESIC, 3, 32-33.

Smith, R.E. 1993. Integrating information from advertising and trial: processes and effects on consumer response to product information. Journal of Marketing Research, 30, 204-19.

Spotts, H. 1994. Evidence of relationship between need for cognition and cronological age: implications for persuasion in consumer research. Advances in Consumer Research, 21, 238-43. 
Te'eni-Harari, T., Lampert S.I. y Lehman-Wilzig S. 2007. Information processing of advertising among young people: the Elaboration Likelihood Model as applied to youth. Journal of Advertising Research, 326-40.

Warrintong, P. y Shim, S. 2000. An empirical investigation of the relationship between product involvement and brand commitment. Psichology \& Marketing, $17,761-82$.

Walsh, G. y Mitchell, V. 2005. Demographic characteristics of consumers who find it difficult to decide. Marketing Intelligence \& Planning, 23, 281-95.

Wu, S.I. 2001. An experimental study on the relationship between consumer involvement and advertising effectiveness. Asia Pacific Journal of Marketing and Logístics, 13, 43-56.

Yoon, C (1997). Age differences in consumer' processing strategies: an investigation of moderating influences, Journal of Consumer Research, 24, 329342.

Yoon, K., Laczniak, R.N., Muehling, D.D. y Reece, B.B. 1995. A revised model of advertising processing: extending the dual mediation hypothesis. Journal of Current Issues and Research in Advertising, 17, 53-67.

Zimbardo, P.G. y Leippe, M.R. (1991). The psychology of attitude change and social influence, New York, McGraw-Hill.

\section{Para citar este artículo:}

Estrada, M - Sánchez, J. - Fandos, J.C. (15-12-2011). EL PROCESAMIENTO DE LA INFORMACIÓN DE LAS PERSONAS MAYORES Y SU ACTITUD HACIA EL ANUNCIO .

REDMARKA - CIECID - Unidad de Investigación en Marketing Aplicado-Universidad de A Coruña

Año IV, Número 7, V1, pp.3-31

ISSN 1852-2300

URL del Documento: cienciared.com.ar/ra/doc.php? $\mathrm{n}=1594$

URL de la Revista: cienciared.com.ar/ra/revista.php?wid=39 